\title{
BEAM PROFILE DATA ANALYSIS FOR THE RHIC INJECTION LINE *
}

\author{
Ping Zhou \\ Bldg 830 \\ Brookhaven National Laboratory \\ Upton, NY 11973
}

\begin{abstract}
Beam profile monitors employing phosphor screens will be used in the beam transfer line from AGS to RHIC, now under construction at BNL, as beam profile monitors. Data analysis procedures are being developed for the transfer line test scheduled for the fall of 1995. In addition to the emittance and Twiss parameters calculation, it will include direct reconstruction of the beam phase space density distribution by using an Abel inversion with the minimal assumption of an elliptically symmetric phase space distribution. The percent emittance comes out of the process naturally.
\end{abstract}

\section{INTRODUCTION}

Two dimensional beam profiles will be measured using the video profile system [1] in the RHIC injection line. They will be used to monitor beam quality and measure beam emittance and Twiss parameters for beamline matching. Six flags will be for emittance measurement, though at most four can be used simultaneously, limited by the number of frame grabbers. This will enable us to measure the input beam parameters on a bunch by bunch basis with some redundancy for error checking.

The information on horizontal and vertical coupling extracted from the two dimensional profile directions will be used to identify and in feedback to correct the coupling. If significant coupling is present a complete four dimensional phase space analysis, ignoring the even more complicated additional coupling with the longitudinal dimension, is necessary. A meaningful and reliable complete phase space analysis is impossible by virtue of the number of flags, the necessary measurement accuracy and the complexity of the required analysis. We will assume the coupling effect is negligible after correction and limit the analysis to one dimension at a time.

\section{SCATTERING EFFECTS}

Going through the flags, beam particles will be scattered. The bulk of the deflection is due to elastic Coulomb scattering from the nuclei within the screen. The distribution of the deflections is roughly Gaussian for small deflections while having greater probability for large-angle scattering[2]. In the limit of thin films where particles' position can be treated as unchanged going through the target, the scattering effect can be easily calculated[3] using a Gaussian scattering angle distribution and a Gaussian beam distribution

$$
\epsilon^{\prime}=\epsilon \sqrt{1+\beta \Theta^{2} / \epsilon}, \quad \alpha^{\prime}=\frac{\alpha}{\sqrt{1+\beta \Theta / \epsilon}}
$$

*Work performed under the auspices of the U.S. Department of Energy

$$
\beta^{\prime}=\frac{\beta}{\sqrt{1+\beta \Theta^{2} / \epsilon}}, \quad \gamma^{\prime}=\frac{\gamma+\Theta^{2} / \epsilon}{\sqrt{1+\beta \Theta^{2} / \epsilon}}
$$

where $\Theta$ is the rms deflection angle going through flag. This result can be extanded to arbitrary distributions for rms quantities.

With the RHIC injection line parameters, in the worst case the emittance dilution can reach $30 \%$ which will greatly affect the accuracy of the measured emittance. Fortunately, scattering effects can be compensated with measurements.

If the beam transfer matrix from flag $i$ to the next flag $i+1$ is $\left(\begin{array}{ll}T_{11} & T_{12} \\ T_{21} & T_{22}\end{array}\right)$ then the increase of rms beam width at flag $i+1$ due to the scattering of flag $i$ follows

$$
\Delta \sigma^{2}=T_{12}^{2} \cdot \Theta^{2}
$$

This effect adds up quadratically from flag to flag. The above equation can be used to obtain each flag's scattering effect by measuring the beam width with and without the flag in front. Thus the effect of scattering on emittance measurement, at least mostly, can be corrected.

\section{EMITTANCE CALCULATION}

At least three independent measurements are needed to determine $\alpha, \beta, \gamma$ and $\epsilon$. The measurements do not not necessarily have to be simultaneous. With a very good repeatability from bunch to bunch, they could also be from measurements of multiple bunches with flags at different locations. They could also be measurements with one or more fixed flags but with varying magnet settings.

Emittance and Twiss parameter calculation from beam width measurements is strait forward[4][5]. Basic formula are reproduced here for reference. Details can be found in [4][5] and [6].

The beam width at flag $i$ is related to the parameters at location $s_{0}$ as $y_{i} \equiv w_{i}^{2}=\beta_{i} \epsilon=\sum_{k} g_{i k} \cdot a_{k}$, where $g_{i 1}=t_{11}^{2}$, $g_{i 2}=-2 t_{11} \cdot t_{12}, g_{i 3}=t_{12}^{2}$ with $\left[t_{i j}\right]$ being the transfer matrix from $s_{0}$ to flag $i$, and $a_{1,2,3}=\epsilon \beta, \epsilon \alpha, \epsilon \gamma$.

Minimizing

$$
\chi^{2}=\sum_{i}\left[\frac{y_{i}-\sum_{k} g_{i k} \cdot a_{k}}{\sigma_{i}}\right]^{2},
$$

where $\sigma_{i}$ is the rms error in $w_{i}^{2}$, yields the normal equation for the problem

$$
\left[N_{j k}\right]\left[a_{k}\right]=\left[b_{j}\right]
$$

where

$$
N_{j k}=\sum_{i} \frac{g_{i j} \cdot g_{i k}}{\sigma_{i}^{2}}, \quad b_{j}=\sum_{i} \frac{g_{i j} \cdot y_{i}}{\sigma_{i}^{2}}
$$


The solution is simply

$$
\left(a_{j}\right)=\left[V_{j k}\right]\left(b_{k}\right)
$$

where $[V]=[N]^{-1}$.

The standard error for any dependent variable, $f=f\left(a_{i}\right)$, can be easily calculated with $[V]$ :

$$
\sigma_{f}^{2}=\sum_{i}\left(\frac{\partial f}{\partial y_{i}}\right)^{2} \sigma_{i}^{2}=\sum_{m, n} \frac{\partial f}{\partial a_{m}} \cdot V_{m n} \cdot \frac{\partial f}{\partial a_{n}}
$$

For $a_{j}$ it is simply $\sigma_{a_{j}}^{2}=V_{j j}$.

The calculations done with artificial profiles for the RHIC injection line show that with $10 \%$ rms error in the measured beam widths, the standard error in calculated beam emittance varies from a few percent to above $20 \%$ depending on which three flags are used, reflecting on the between-flag betatron phase advances. When two flags are multiples of $180^{\circ}$ of betatron phase apart they are images of each other and do not provide independent data. When the phase advance is close to multiples of 180 degrees, theoretically the problem is perfectly solvable, but the result is increasingly sensitive to small errors in beam widths. The phase advance, however, depends on initial beam parameters as well as the transfer line lattice, so what actually turns out in actual measurements may be different from bunch to bunch.

\section{PHASE SPACE DISTRIBUTION}

The full property of the beam is characterized by its phase space density distribution. Once the Twiss parameters and emittance are determined, this phase space distribution can be reconstructed from the measured density using Abel transformation technique, and the only underlining assumption needed is that the distribution has elliptical symmetry. Having the 2dimensional phase space distribution also makes the calculation of partial beam emittance easy and unambiguous. There have been percentage emittance calculations reported with various assumption[5][4]. Elliptical symmetry is the least assumptions needed for this purpose.

A 2-dimensional beam phase space distribution with elliptical symmetry, $\rho\left(x, x^{\prime}\right)=\rho\left(\gamma x^{2}+2 \alpha x x^{\prime}+\beta x^{\prime 2}\right)$, can be transformed into a circular distribution in $(u, v)$ space with the transformation

$$
\left(\begin{array}{l}
u \\
v
\end{array}\right)=\frac{1}{\sqrt{\beta}}\left(\begin{array}{ll}
1 & 0 \\
\alpha & \beta
\end{array}\right)\left(\begin{array}{l}
x \\
x^{\prime}
\end{array}\right)
$$

The resulting distribution will be $\rho\left(u^{2}+v^{2}\right)$. The projection of the phase space distribution is directly related with the measured profile by

$$
\int \rho\left(x, x^{\prime}\right) d x^{\prime}=\frac{1}{\sqrt{\beta}} \int \rho(u, v) d v
$$

Now $\rho(u, v)$ has circular symmetry, i.e. $\rho(u, v)=\rho\left(u^{2}+\right.$ $v^{2}$, and the distribution can be recovered from the projection through inverse Abel transform[7].

For a function with circular symmetry in $(x, y)$ space, $f(x, y)=f(r)$, Abel transforms establish the relation between the distribution and its projections:

$$
\begin{aligned}
f_{A}(x) & =\int_{-\infty}^{\infty} f(r) d y=2 \int_{x}^{\infty} \frac{f(r) r d r}{\sqrt{r^{2}-x^{2}}} \\
f(r) & =-\frac{1}{\pi} \int_{r}^{\infty} \frac{f_{A}^{\prime}(x) d x}{\sqrt{x^{2}-r^{2}}}
\end{aligned}
$$

The direct inversion in Eq. 1 involves derivation and integration of a divergent function and therefore is not suited for numerical applications. There are various ways to get around the problem[8], including the filtering of raw data to reduce noise, using transform techniques to avoid the divergence. One can also fit the data to analytical functions based on physical models and carry out the inversion analytically. The latter approach is what we have chosen for its easy control. It reduces the further computation and also serves the noise filtering purpose.

We choose Gaussian weighted Hermite polynomials to expand the beam profile:

$$
\rho(x)=\sum_{n} h_{n} H_{n}\left(\frac{x}{\sigma}\right) e^{-\frac{1}{2}\left(\frac{x}{\sigma}\right)^{2}}
$$

Because this set of functions are orthogonal, we can avoid the fitting of parameters thus simplifying the calculation of expansion coefficients. Since the undistorted profile should be symmetric about the center, based on the elliptical phase space distribution assumption, we only need the even orders of Hermite polynomials. The maximum order that has to be used in the expansion, however, is not as small as we might have expected or hoped, even when the profile is close to a Gaussian distribution. This is because errors in the calculation of rms of the profile will introduce many high order components. As a result 10 to 20 terms have to be included usually. Thankfully, the expansion process itself is very fast and high number of terms does not constitute much inefficiency in the overall reconstruction process.

The Abel inversion of the Gaussian weighted Hermite polynomials can be calculated analytically[3]. The values of the functions, their Abel inversions and the fraction of beam inside any radius can all be calculated through recursion relations. The result of the inversion is in the form of

$$
\rho(r)=\sum_{n} a_{n} r^{n} e^{-\frac{r^{2}}{2}} .
$$

where $r$ is normalized to the beam rms size.

The reconstructed phase space distribution, with normalized coordinate against the rms beam size, can be directly used to calculate the percentage emittance, or the corresponding beam profile through Abel transform.

The fraction of beam inside a radius $\mathrm{R}$ in the $(u, v)$ normalized phase space, is

$$
\delta(R)=2 \pi \int_{0}^{R} \rho(r) r d r
$$

Since $R$ is normalized against rms beam size, the percentage emittance corresponding to $\delta$ is simply

$$
\epsilon_{\delta}=R \epsilon
$$




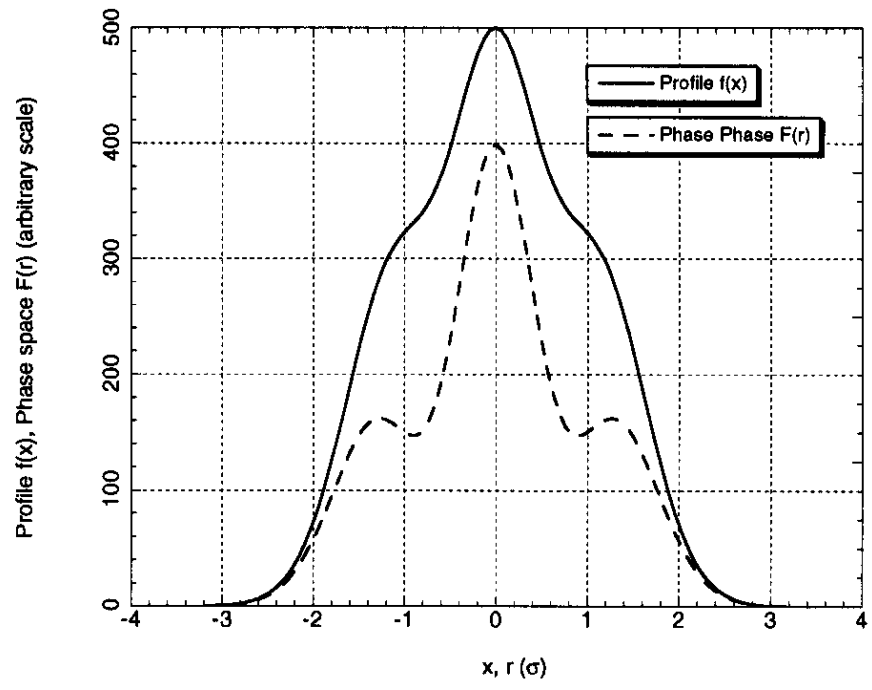

Figure 1. Beam profile and reconstructed phase space distribution.

The fractional beam profile, that of the fraction in the central part of the phase space can also be obtained by Abel transforming $\rho(r)$ with its value set to zero outside $r=R$.

As an example, a beam profile composed of a Gaussian plus a shoulder component is constructed and its corresponding phase space distribution is calculated using the technique described above. They are shown in Fig. 1. The profiles for different fractions of core beam can also be easily extracted. They are shown in Fig. 2 for the example above.

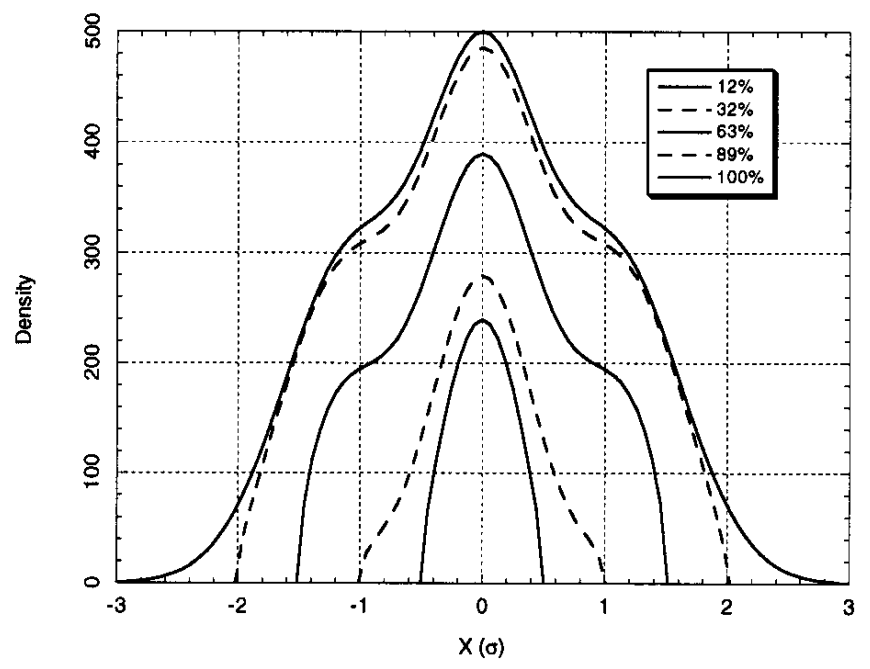

Figure 2. Profiles of various core beam fractions.

In the case where elliptical symmetry assumed throughout this report is lost, the reconstruction of phase space distribution theoretically need infinite number of profile measurements. Except the aspect ratio change that can happen in the beam phase space, the problem is the same as in computed tomography. To reconstruct a 2-dimensional object from all of its projections is the 2-dimensional Radon inversion problem. With the very limited number of beam profiles we can only expect to extract some coarse features of the phase space distribution using some of the numerical techniques in doing Radon inversion. This has yet to be investigated.

\section{References}

[1] R.L. Witkover. Design of the beam profile monitor system for the rhic injection line. These proceedings.

[2] T. G. Trippe. Physics Vade Mecum, chapter 9, page 160. American Institute of Physics, 2nd edition, 1989.

[3] P. Zhou. Beam profile monitor data analysis. Internal Report RHIC/AP/43, BNL, 1994.

[4] H. Ploss and L. N. Blumberg. Methods of emittance measurement in external beams using ellipse approximations. Internal Report AGS DIV 68-4, BNL, 1968.

[5] Kiyokazu Ebihara, et al. Non-destructive emittance measurement of a beam transport line. Nuclear Instruments and Methods, 202:403-409, 1982.

[6] Willianm H. Press, et al. Numerical Recipes in C, chapter 15. Cambridge University Press, 2nd edition, 1992.

[7] R. N. Bracewell. The Fourier Transform and Its Applications, chapter 12. McGraw-Hill, 2nd edition, 1986.

[8] L. M. Smith, et al. Abel inversion using transform techniques. Journal of Quantitative Spectroscopy and Radiative Transfer, 39(5):367-373, 1988. 